\title{
Automatic Diagnosis of Diabetic Retinopathy Micro aneurysm from Low Contrast Retinal Images using Mathematical Morphology Methods
}

\author{
Raju Maher \\ Research Scholar \\ Department of \\ Computer Science \& IT, \\ Dr. Babasaheb \\ Ambedkar \\ Marathwada University, \\ Aurangabad, India
}

\author{
Sangramsing Kayte \\ Research Scholar \\ Department of \\ Computer Science \& IT, \\ Dr. Babasaheb \\ Ambedkar \\ Marathwada University, \\ Aurangabad, India
}

\author{
Dnyaneshwar \\ Panchal \\ Research Scholar \\ Department of \\ Computer Science \& IT, \\ Dr. Babasaheb \\ Ambedkar \\ Marathwada University, \\ Aurangabad, India
}

\author{
Suvarnsing G. \\ Bhable \\ Research Scholar \\ Department of \\ Computer Science \& IT, \\ Dr. Babasaheb \\ Ambedkar \\ Marathwada University, \\ Aurangabad, India
}

\author{
Pankaj Sathe \\ Research Scholar \\ Department of Computer Science \& IT, Dr. \\ Babasaheb Ambedkar Marathwada University, \\ Aurangabad, India
}

\author{
Jaypalsing Kayte \\ Research Fellow \\ Department of Computer Science \& IT, Dr. \\ Babasaheb Ambedkar Marathwada University, \\ Aurangabad, Indi
}

\begin{abstract}
Diabetic retinopathy is a disease of the retina, occurring in about a quarter of people with diabetes. The retina contains cells that convert the light into the electric signals, and these signals are then sent on to the brain. The symptoms can blur or distort the patient's vision and are a main cause of blindness. Microaneurysm are one of the primary signs of retinopathy. Detection of exudates by ophthalmologists normally requires pupil dilation using a morphological method which takes time and affects patients. The leakage causes the retina to swell up a little and become waterlogged, a bit like a sponge. This swelling then damages the retinal cells themselves. This paper examines and proposes a set of optimally adjusted morphological operators to be used for Microaneurysm detection on diabetic retinopathy low-contrast images. Automatic tool for diagnosis of diabetic retinopathy must go through some well-defined steps. First, it has to detect the blood vessels and optic disc. Second, it has to identify abnormalities in the retina like exudates and microaneurysms that cause diabetic retinopathy. This method has resulted with sensitivity and specificity for our Microaneurysm detection is $80 \%$ and $99.5 \%$, respectively.
\end{abstract}

\section{Keywords}

Diabetic retinopathy; Microaneurysm; Morphology operator; Retinal image; Non-dilated retinal images.

\section{INTRODUCTION}

Diabetes is the commonest cause of blindness in the working age group in the developed world. Diabetic retinopathy (DR) is caused by damage to the small blood vessels of the retina in the posterior part of the eye of the diabetic patient. DR is an eye disease and a common complication of diabetes that can cause vision loss if left undiagnosed at an initial stage. It is the prime cause of blindness in the working age population of the world. The screening of diabetic patients for the development of diabetic retinopathy can potentially reduce the risk of blindness in the patients by $50 \%$ [1-3].
Diabetes is a chronic disease that is reaching epidemic proportions worldwide. There are currently more than 190 million people with diabetes worldwide. The World Health Organization (WHO) estimates that this will rise to 221 million by the year 2010, largely due to population growth, ageing, urbanization and a sedentary lifestyle. Diabetic retinopathy is the most normal cause of new cases of blindness among adults aged $20-70$ years. During the first two periods of disease, nearly all patients with type 1 diabetes and greater than $90 \%$ of patients with type 2 diabetes have retinopathy [1]. Exudates occur when they are the lipid residues of serous leakage from damaged capillaries. The commonest cause is diabetes. The number of microaneurysms, haemorrhages and exudates increases as the degree of disease [4][25]. A number of techniques for microaneurysm and haemorrhage detection have been proposed. Sinthanayo thinetal.[5]applied recursive region growing segmentation (RRGS) technique to segment vessels, microaneurysms and haemorrhages. The yellow-white flecks scattered around the macular region are little mounds beneath the retinal pigment epithelium. They are tombstones of dead retinal pigment epithelial cells. The commonest cause is agerelated macular degeneration, a common but poorly understood disorder of the elderly. At this stage, visual acuity may be only slightly abnormal. If the retinal pigment epithelium dies further, vision will fail as the foveal region turns into a confluent yellow-white area. The gray portion lies under the retinal pigment epithelium. Surrounding the gray hemorrhage is a ring of red blood that has escaped into the retina. This patient has age-related macular degeneration, the commonest cause of choroidal neovascularization and submacular hemorrhage. Then the detected red lesion candidates were classified using a number of features and a knearest neighbour classifier. Usher et al. [7][24] used an RRGS, adaptive intensity thresholding and edge enhancement operator to extract the candidate red lesions. Candidate red lesions were classified using a neural network. However, in this paper we concentrate on exudate detection as a visible sign of diabetic retinopathy and a marker for the presence of 
coexistent retinal edema. If the exudates extend into the macular area, vision loss can occur. Fluorescein angiogram images provide important information on pathologies. The most effective and accurate ways to ask that authors follow some simple guidelines. In essence, we ask you to make your paper look exactly like this document. The easiest way to do this is simply to download the template, and replace the content with your own material.

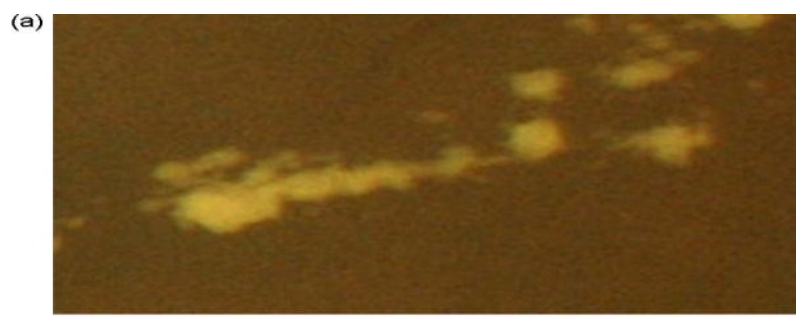

(b)

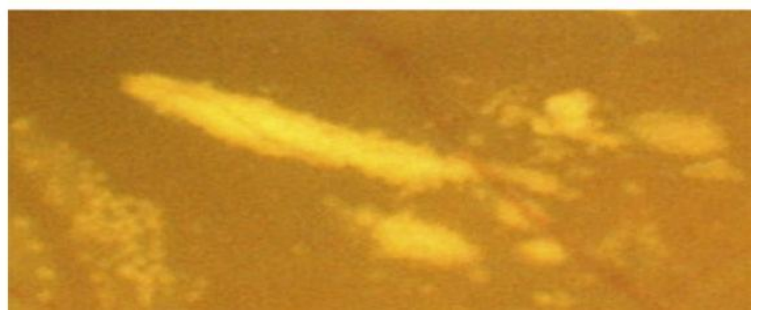

Fig. 1. (a) And (b) Retinal image containing exudates.

observe and diagnose diabetic macular edema are to investigate a fluorescein angiography. In practical terms, the decision whether to laser treat the retina does not depend significantly on the images from fluorescein angiography, it is mostly done without this investigation. The fluorescein angiograms are not suitable for an automatic screening system because there are side-effects associated with giving a patient fluorescein. The use of colour fundus images is more suitable for an automatic screening system. An automatic exudate detection system would be useful in order to detect and treat diabetic retinopathy in an early stage. This blood vessels on the retinal surface is a crude attempt at vascularizing ischemic tissue. Retinal neovascularization lacks the bifurcating pattern of normal vessels. Poorly and hastily built, they bleed spontaneously or with minimal trauma. The blood pours into the retina and into the interface between retina and vitreous, where it attracts fibroglial elements which form fibro vascular stalks. The stalks induce vitreous contraction which pulls them farther away from the retina. Eventually the retina detaches. The combination of hemorrhage and retinal detachment spells blindness. The common causes of retinal neovascularization on or near the optic disc are diabetes, retinal vein occlusion, and radiation. Neovascularization in the peripheral retina (out of view of the direct ophthalmoscope) is most commonly caused by sickle cell disease and retinopathy of prematurity. as shown in Fig. 1(a) and ( $b$ ). The thresholding and RRGS technique were widely used. Sinthanayothin et al. [5] reported the result of an automated detection of diabetic retinopathy on digital fundus images by RRGS algorithm where the performance was measured on $10 \times 10$ patches rather on the whole image. Usher et al. [7] detected the candidate exudates region by using a combination of RRG Sandadaptiveintensity thresholding.Thecandidate regions were extracted and used as input to a neural network. Poor quality images affected the separation result of bright and dark lesions using thresholding and exudate feature extraction using RRGS algorithm. Zheng et al. [9][22] detected exudates using thresholding and a region growing algorithm. The fundus photographs were taken with a non-mydriatic fundus camera and were then scanned by a flat-bed scanner.

Colour normalization and local contrast enhancement followed by fuzzy C-means clustering and neural networks were used by Osareh et al. [10]. The system works well only on Luv colour space but in the case of non-uniform illumination the detection accuracy is low. Mitra et al. [11] applied naïve Bayes classifier for diagnosis of diseases from retinal image. A system can provide a good decision support to ophthalmologist. Most techniques mentioned earlier worked on dilated pupils in which the exudates and other retinal features are clearly visible. Based on experimental work reported in previous work, good quality images with larger fields are required. The retinal image of the patient must be clear enough to show retinal detail. Low quality images (non-uniform illumination, low contrast, blur or faint image) do not perform well even when enhancement processes were included. The examination time and effect on the patient could be reduced if the system can succeed on nondilated pupils. Furthermore, many techniques required intensive computing power for training and classification. This paper proposes an exudate detection techniques based on mathematical morphology on retinal images of non-dilated pupils that are low quality images. We based our work on this technique because it is very fast and requires lower computing power. So that the final system can be used even on a very poor computer system, such as those that may be available in rural area in developing countries where both expert ophthalmologists and high performance computers are rarely available. In addition, the location of exudates based on macular position is important information for an ophthalmologist $[12,13]$. They show the severity of disease, where exudates that appear closer to the macular indicate an increased severity of disease. A grid circle centred on the macular was added to provide improved diagnosis to the ophthalmologist.

\section{METHODS}

All digital retinal images were taken from DIARETDB1. Histogram equalization in which manipulates histograms in a consistent and meaningful manner. DIARETDB1 images were resized to $340 \times 480$ pixels in order to reduce the processing time of the method However, many image enhancement techniques are based on spatial operations performed on local neighbourhoods. The detection method proposed in this paper is performed in MATLAB (version 2012). The accuracy of the method was tested in the public database of fundus images DIARETDB1 database consists of 89 colour fundus images of which 84 contain at least mild non-proliferative signs of the diabetic retinopathy and five are considered as normal which do not contain any signs of the diabetic retinopathy according to all the experts participated in the evaluation. Images were captured with the same 50 degree FOV digital fundus camera with varying imaging controlled by the system in the Kuopio university hospital, Finland. The image ground truth provided along with the database is based on expert selected findings related to the diabetic retinopathy and normal fundus structures[25].

\subsection{Pre- processing}

In pre-processing, we create binary masks for background and noisy areas. Different retinal images make it difficult to extract retinal features and distinguish exudates from other contrast, and brightness bright features in images. colour components. A median filtering operation was then applied on I band to reduce noise before a contrast-limited adaptive histogram equalization was applied for contrast enhancement 
[14]. CLAHE operates on small regions in the image. The contrast of each small region is enhanced with histogram equalization. After performing the equalizations, the neighbouring small regions were then combined by using bilinear interpolation. Exudate lesions and optic disc regions normally show high intensity values in this channel and thus the contrast enhancement technique assigns them the highest intensity values $[10,15]$.

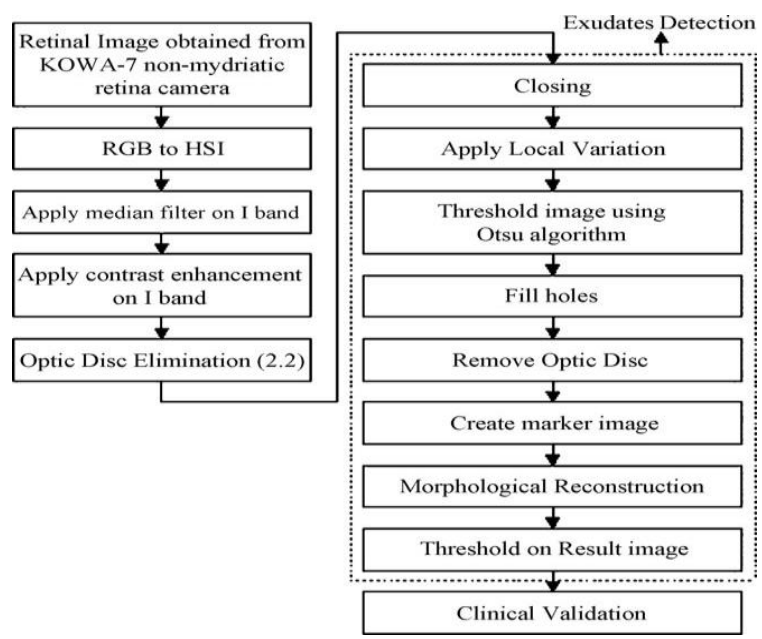

Fig. 2. Procedure of the proposed exudates detection.

\subsection{Optic disc elimination}

$$
\begin{aligned}
& J_{q}(U, V)=\sum_{k=1}^{n} \sum_{i=1}^{c}\left(u_{i k}\right)^{q} d^{2}\left(x_{k}, v_{i}\right) \\
& \text { Where } x=\left\{x_{1}, x_{2}, \ldots x_{k}\right\} \subseteq r^{p},
\end{aligned}
$$

The optic disk is characterized by the biggest high-contrast area. The optic disk is roughly detected by using the entropy feature on the contrast enhanced image. The entropy is a measure of randomness a window of size $9 \times 9$ pixels is used. The resulting image is threshold using svm algorithm in order to eliminate the regions with low local variation. To include the neighboring pixels of the threshold result, a dilation operator is used. A flat disk shaped structuring As a result, high intensities are reconstructed while the rest is removed, as shown in Fig. 3( e ). Normally, the optic disc can be easily identified as the largest area. Element having radius However, in some cases such as the appearance of huge exudates in the image, there might be some areas in the image which are larger than the optic disc. Because the shape of optic disc is round, therefore the optic disc region selection process needs to be made specific to the largest one among the regions whose shapes are circular of eleven is employed for dilation. The eliminated optic disk regions [20].
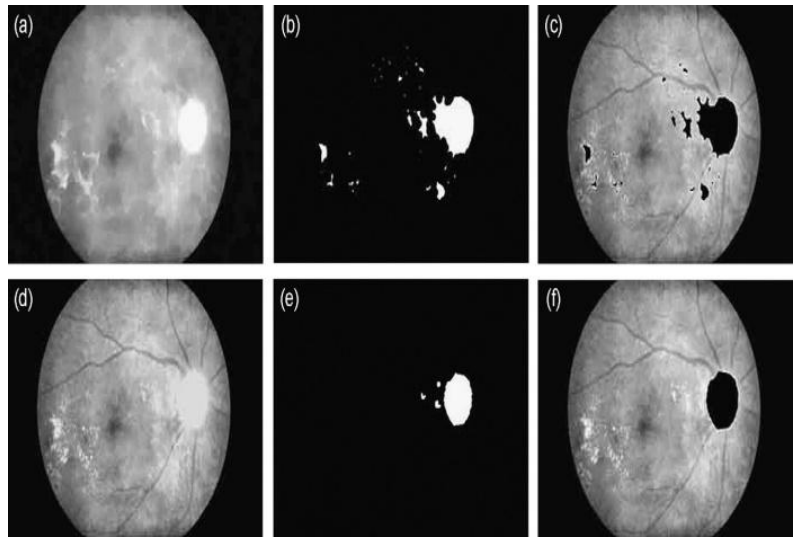

Fig. 3. (a) Intensity image after closing, (b) thresholded image, (c) marker image, (d) reconstructed image, (e) thresholded result of difference image and (f) optic disc area eliminated from the contrast enhanced image.

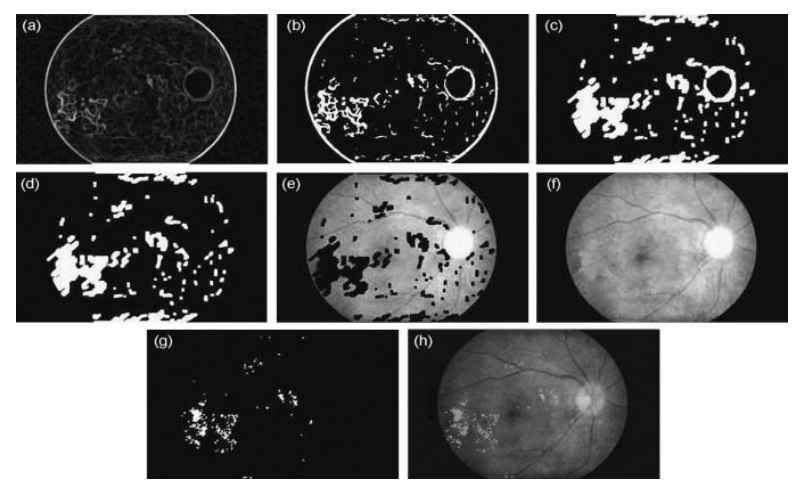

Fig. 4. Exudates detection of "image 1". (a) Local variation image, (b) thresholded image, (c) enclosed areas were flood-filled, (d) optic disc was removed from image, (e) marker image, (f) reconstructed image, (g) difference image AND (h) result superimposed on the original image.

The selected result (largest among circular shapes), OP5, was dilated with a binary dilation operator $(\delta)$ in Eq. (5) to ensure that all pixels in the optic disc area are covered. This step, a flat disc-shaped structuring element with a fixed radius of six (B2) is used.

All optic disc area in the original image was masked out using the previous output.

\subsection{Exudate detection}

Exudates that is bigger than the linear structuring element cannot be identified. If the length of the structuring element is increased to extract larger objects, then more spurious candidate exudates objects will be detected on blood vessels as the segmentation of blood vessels deteriorates. A pixel classification based method can be used to eliminate this drawback. By combining blood vessel segmentation method presented in Chapter-3, with exudates detection, larger candidates can be extracted and the number of spurious candidates on the vessels will be reduced. In this method, both the blood vessels and candidate exudates are segmented at once and later the blood vessels are separated from the candidate microaneurysms. This approach comprises two steps. Firstly matched filtering is applied to enhance the dark objects like blood vessels and exudates against background.

$E_{2}(x)=\frac{1}{N-1} \cdot \sum_{i \in W(x)}\left(E_{1}(i)-\mu_{E_{1}}(x)\right)^{2}$ 
where $\mathrm{x}$ is a set of all pixels in a sub-window $\mathrm{W}(\mathrm{x}), \mathrm{N}$ is a number of pixels in $\mathrm{W}(\mathrm{x}), \mu \mathrm{E} 1(\mathrm{x})$ is the mean value of $\mathrm{E} 1(\mathrm{i})$ and $i \in W(x)$. A window size of $7 \times 7$ was used in this step. Using matched filtering to get rid of all regions with low local variation. To ensure that all the neighbouring pixels of the thresholded result were also included in the candidate region, a binary dilation operator was also applied with a flat discshaped structuring element with a fixed radius of six (B3), as indicated in Eq. (7). The result is shown in Fig. 4( b ). The matched filter detection [18] is applied to detect exudates in fundus images. exudates usually have poor local contrast. A two-dimensional matched filter kernel is used to convolve with the preprocessed fundus image to enhance the contrast exudates. therefore the enclosed area was flood-filled. The image is represented by E4, and the result is shown.

$$
\text { Sensitivity }=\frac{\mathrm{TP}}{\mathrm{TP}+\mathrm{FN}} \times 100
$$

Specificity is defined as the percentage of normal images classified by the system Accuracy in percentage is the measure of

$$
\text { Specificity }=\frac{T N}{T N+F P} \times 100
$$

Accuracy is the percentage of correctly classified normal and abnormal images.

$$
\text { Accuracy }=\frac{\mathrm{TP}+\mathrm{TN}}{(\mathrm{TP}+\mathrm{FN}+\mathrm{TN}+\mathrm{FP})} \times 100
$$

The performance of the proposed algorithm is tested in terms of True positive detections (TP), (TN) true negative detections, (FP) false positive detections, (FN) false negative detections, (ToP) total positives (manually counted from the image), (MP) missed points, (PR) precision (accuracy), (FR) false activation rate, (TR) true positive rate, $(\mathrm{SN})$ Sensitivity, false activation rate. True positive (TP) indicates that the patient suffers from the disease and test result was also positive, false positive (FP) indicates that the patient does not suffer from the disease and was diagnosed as positive, true negative (TN) indicates that the patient does not suffer from the disease and was diagnosed as negative, false negative (FN) indicates that the patient suffers from the disease but diagnosed as negative.

\section{RESULTS}

89 images were tested on an dual core $1.25 \mathrm{GHz}$ PC using MATLAB 2012. The result of the exudates detection was superimposed on the original image. Images were captured using the same $150 \circ$ FOV digital fundus camera with varying imaging settings. Figures 2 the output of classifier highlighting dark and bright lesions from retinal images respectively. The optic disc was also detected well and removed. Usually there are no exudate pixels around the optic disc so the removal of the optic disc did not affect the exudate detection[24],[25].

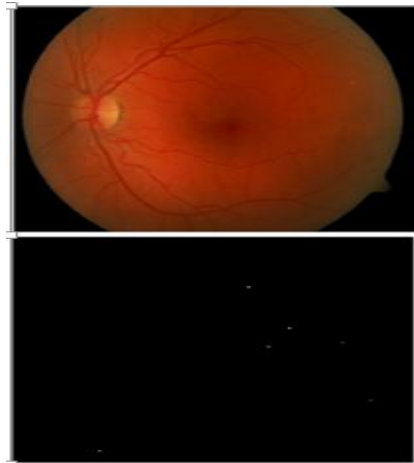

(A)

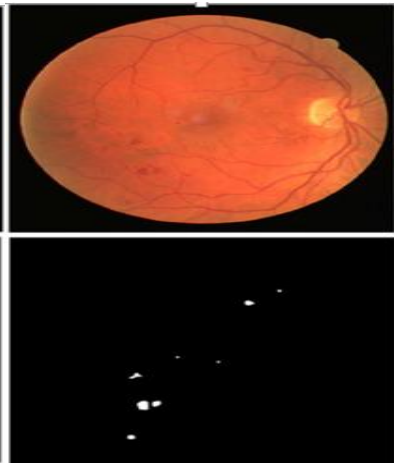

(B)

\section{Fig.5. (a) Normal Image $\quad$ (b) Exudate (DR) image}

The performance of proposed technique was evaluated quantitatively by comparing the resulting extractions with ophthalmologists' images pixel by pixel. A total of 30 images are selected from all two databases at random and for those images, a comparison between proposed method and ground truth for lesion. The performance of the proposed system is evaluated on the basis of four measures, namely, True positive (TP) indicates that the patient suffers from the disease and test result was also positive, false positive (FP) indicates that the patient does not suffer from the disease and was diagnosed as positive, true negative $(\mathrm{TN})$ indicates that the patient does not suffer from the disease and was diagnosed as negative, false negative (FN) indicates that the Sensitivity and specificity were chosen as our measurement of accuracy of the algorithms at the pixel level. Notonly does this evaluation mechanism show how accurate our detection was, it also shows how inaccurate our detector can be. This pixel-based evaluation considers four values, namely true positive (TP After all the 40 retinal images with exudates and 20 normal retinal images without exudates were processed, they were compared with the hand-drawn ground-truth images. Table 1 shows the quantitative result of TP, FP, FN, TN, sensitivity, specificity and misclassified proportion from the images of diseased eyes. For our data set with diabetic retinopathy exudates, the sensitivity and specificity of the exudate detection are $80 \%$ and $99.5 \%$, respectively. For normal retinal image detection, the specificity and misclassified proportion are $99.9 \%$ and $7.2 \%$, respectively. The sensitivity cannot be calculated in which TP and FN values are all zero due to no exudates in ground-truth images. Proposed algorithm has very high specificity which show that the algorithm does not recognize a non-exudate pixel as an exudate pixel. However, the sensitivity, a relatively lower value, also showed that the low intensity exudates pixels are still too elusive to be detected by this algorithm. The system also detects the macular region in order to provide the ophthalmologists with the distance information between the detected exudates and the macular. The exudates with in the inner circle will affect the vision of patients more than the ones outside it. As shown in Fig. 3(a), exudates are present nearer to the macular than exudates inFig. 3 (b).

\section{DISCUSSION}

In this work we have investigated and proposed a set of optimally adjusted morphological steps to automatically detect optic disc and exudates from diabetic retinopathy patient's non-dilated pupil digital images in an attempt to detect the pathologies earlier. The exudate detection because the intensity features of both areas are similar. From 20 normal patients, the misclassified proportion value of seven people is over the average misclassified proportion and might 
need a further diagnosis. It means that this system can reduce the ophthalmologist's workload especially in developing countries where the number of ophthalmologists is not sufficient to deal with the large number of diabetic retinopathy patients. This system intends to help the ophthalmologists in the diabetic retinopathy screening process for detecting the symptoms faster and more easily. The results demonstrated here indicate that automated diagnosis of diabetic retinopathy based on intensity retinal image analysis can be very successful in detecting exudates. It is not a final result application but it can be a preliminary diagnosis tool or decision support system for ophthalmologists.

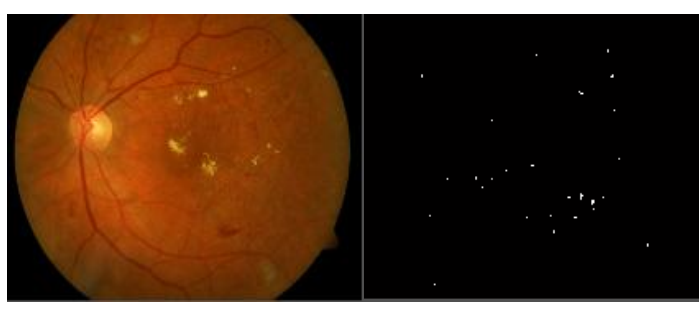

Fig. 6. (a) RGB image with Microaneurysm (b) after applying filter detection result of microaneurysm[22][23]

Human ophthalmologists are still needed for the cases where detection results are not very obvious.

There are also some incorrect exudate detections which are the noise in the image acquisition process, the exudates that are proximate to blood vessels or because the exudates appear very faint. There are also some incorrect exudate-like detections on a normal retinal image as shown in Fig. 5(b). Strong and high contrast choroidal blood vessels, which appear and lie in the retina background are incorrectly detected as exudates. The missing faint exudates may have not affected the sensitivity much since even human experts are not sure about some ambiguous regions. However, the performance of the algorithm can be improved if these set of low-contrast exudates can be detected. We might have to add more specific features to the system in order to get these kinds of regions to be detected in the future.

\section{CONCLUSION AND FUTURE WORK}

Automated system for diabetic retinopathy detection has been presented. This method was developed to detect exudates from low-contrast, retinal digital images of retinopathy images an In this work we have investigated and proposed a computer-based system extracts blood vessels and exudates for grading the severity of the diabetic retinopathy to identify normal, NPDR and PDR. Early detection of diabetic retinopathy is very important because it enables timely treatment that can ease detect bright objects Sharply with an average sensitivity $95.4 \%$. The experimental results show that the proposed method yields better sensitivity and predictive values compared to other methods. Therefore, one of the major strengths of the proposed system is accurate feature extractions and accurate grading of NPDR lesions. In this research image processing components required to build an automated system for early detection of diabetic retinopathy. Future work will address an issue of improving the sensitivity by improving the results of other tasks, such as the detection of the optic disc and blood vessels, and also try to localize faint and small exudates. Infuture, in work system to recognize microaneurysms and haemorrhages, there may be a problem edge filter and feature selection. It is intended that these features will be used in future detection algorithms.

Table 1 the exudates detection results of diseased retinal images

\begin{tabular}{|c|c|c|c|c|c|c|c|c|}
\hline $\begin{array}{l}\text { Digital } \\
\text { images }\end{array}$ & $\begin{array}{l}\text { Ground- } \\
\text { truth } \\
\text { exudates } \\
\text { numbers }\end{array}$ & $\begin{array}{l}\text { Detected } \\
\text { exudates } \\
\text { numbers }\end{array}$ & TP & $\mathbf{F P}$ & FN & $\mathbf{T N}$ & Sensitivity (\%) & Specificity (\%) \\
\hline Image 1 & 278 & 25 & 3,575 & 2,853 & 2,340 & 413 & 71.25 & 98.95 \\
\hline Image 2 & 152 & 195 & 958 & 429 & 214 & 732 & 81.74 & 99.88 \\
\hline Image 2 & 243 & 192 & 231 & 501 & 149 & 799 & 32.33 & 99.87 \\
\hline Image 4 & 448 & 440 & 188 & 831 & 119 & 918 & 31.24 & 99.77 \\
\hline Image 5 & 1333 & 447 & 1,150 & 752 & 202 & 981 & 79.15 & 99.80 \\
\hline Image 3 & 133 & 127 & 1,082 & 1,415 & 158 & 2,222 & 87.27 & 99.32 \\
\hline Image 7 & 119 & 409 & 1,300 & 1,292 & 279 & 373 & 85.15 & 99.32 \\
\hline Image 8 & 113 & 304 & 154 & 884 & 108 & 882 & 58.78 & 99.73 \\
\hline Image 9 & 223 & 181 & 1,352 & 1,450 & 290 & 425 & 80.91 & 99.31 \\
\hline Image 10 & 30 & 359 & 425 & 413 & 94 & 791 & 81.89 & 99.89 \\
\hline Image 11 & 244 & 383 & 521 & 732 & 121 & 405 & 81.15 & 99.80 \\
\hline
\end{tabular}




\begin{tabular}{|c|c|c|c|c|c|c|c|c|}
\hline Image 12 & 137 & 198 & 112 & 799 & 22 & 429 & 77.78 & 99.79 \\
\hline Image 12 & 333 & 739 & 871 & 918 & 283 & 501 & 39.29 & 99.73 \\
\hline Image 14 & 1357 & 407 & 279 & 981 & 280 & 831 & 57.51 & 99.74 \\
\hline Image 15 & 1210 & 511 & 8,782 & 2,222 & 1,888 & 752 & 82.21 & 99.09 \\
\hline Image 13 & 1497 & 211 & 713 & 373 & 5 & 1,415 & 99.21 & 99.82 \\
\hline Image 17 & 242 & 277 & 998 & 882 & 225 & 1,292 & 80.94 & 99.73 \\
\hline Image 18 & 490 & 271 & 227 & 425 & 157 & 884 & 38.22 & 99.89 \\
\hline Image 19 & 1545 & 340 & 258 & 791 & 174 & 1,450 & 59.72 & 99.79 \\
\hline Image 20 & 500 & 1112 & 75 & 405 & 24 & 413 & 38.81 & 99.89 \\
\hline Image 21 & 203 & 304 & 2,835 & 2,413 & 818 & 429 & 77.79 & 99.25 \\
\hline Image 22 & 513 & 222 & 12,780 & 4,333 & 2,777 & 501 & 82.15 & 98.71 \\
\hline Image 22 & 205 & 231 & 2,271 & 1,827 & 745 & 831 & 81.45 & 99.51 \\
\hline Image 24 & 222 & 421 & 2,745 & 1,892 & 320 & 752 & 81.22 & 99.49 \\
\hline Image 25 & 982 & 733 & 3,240 & 4,091 & 1,424 & 1,415 & 81.55 & 98.89 \\
\hline Image 23 & 292 & 790 & 953 & 441 & 173 & 1,292 & 84.45 & 99.88 \\
\hline Image 28 & 914 & 285 & 425 & 1,093 & 91 & 884 & 82.70 & 99.71 \\
\hline Image 29 & 21 & 244 & 24,279 & 8,122 & 1,853 & 1,450 & 92.90 & 97.38 \\
\hline Image 20 & 213 & 99 & 24,845 & 3,311 & 2,457 & 413 & 90.97 & 98.04 \\
\hline Image 21 & 2 & 272 & 3,880 & 4,412 & 820 & 732 & 89.25 & 98.80 \\
\hline Image 22 & 11 & 3 & 13,252 & 2,374 & 1,520 & 799 & 91.44 & 98.97 \\
\hline Image 22 & 44 & 34 & 2,720 & 2,397 & 721 & 752 & 78.82 & 99.28 \\
\hline Image 24 & 72 & 110 & 2,423 & 2,701 & 327 & 1,415 & 84.22 & 99.27 \\
\hline Image 25 & 253 & 48 & 7,712 & 1,893 & 1,428 & 1,292 & 84.29 & 99.48 \\
\hline Image 23 & 24 & 81 & 2,132 & 1,132 & 124 & 884 & 94.58 & 99.39 \\
\hline Image 27 & 145 & 27 & 10,188 & 2,783 & 815 & 1,450 & 92.59 & 99.24 \\
\hline Image 28 & 58 & 84 & 2,590 & 1,211 & 321 & 413 & 80.33 & 99.38 \\
\hline Image 29 & 42 & 114 & 279 & 1,053 & 79 & 732 & 82.75 & 99.72 \\
\hline Image 40 & 123 & 252 & 752 & 1,821 & 98 & 799 & 88.47 & 99.51 \\
\hline Average & 383 & 300 & 3680 & 1,760 & 616 & 875 & 80.00 & 99.43 \\
\hline
\end{tabular}




\section{REFERENCES}

[1] World Diabetes, A newsletter from the World Health Organization, 4, 1998.

[2] Hove MN, Kristensen JK, Lauritzen T, Bek T. Quantitative analysis of retinopathy in type 2 diabetes: dentification of prognostic parameters for developingvisuallosssecondarytodiabeticmaculopathy.Ac taOphthalmol Scand 2004;82:379-85.

[3] Hsu W, Pallawala PMDS, Mong Li Lee, Kah-Guan Au Eong. The role of domain knowledge in the detection of retinal hard exudates. In: Proceedings of the International Conference on Computer Vision and Pattern Recognition IEEE Computer Society, vol. 2. 2001. p. II-243-51.

[4] Kanski J. Diabetic retinopathy, clinical ophthalmology. Oxford: Butterworth-Heimann; 1997.

[5] Sinthanayothin C, Boyce JF, Williamson TH, Cook HL, Mensah E, Lal S. Automated detection of diabetic retinopathy on digital fundus image. J Diabet Med 2002;19:105-12.

[6] Niemeijer M, van Ginneken B, Staal J, Suttorp-Schulten MS, Abramoff MD.Automaticdetectionofredlesionsindigitalcolorfundus photographs.

[7] IEEE Trans Med Imag 2005;24:584-92.

[8] Usher D, Dumskyj M, Himaga M, Williamson TH, Nussey S, Boyce J. Automated detection of diabetic retinopathy in digital retinal images: a tool for diabetic retinopathy screening. Diabet Med 2004;21:84-90.

[9] Gardner GG, Keating D, Williamson TH, Elliott AT. Automatic detection of diabetic retinopathy using an artificial neural network: a screening tool. $\mathrm{Br} \mathrm{J}$ Ophthalmol 1993;80:940-4.

[10] ZhengLiu,OpasC,KrishnanSM.Automaticimageanalysiso ffundusphotograph. In: Proceedings of the International Conference on Engineering in Medicine and Biology, vol. 2. 1997. p. 524-5.

[11] Osareh A, Mirmehdi M, Thomas B, Markham R. Automated identification of diabetic retinal exudates in digital colour images. Br J Ophthalmol 2002;87:1220-2.

[12] Mitra SK, Te-Won Lee, Goldbaum M. Bayesian network based sequential inference for diagnosis of diseases from retinal images. Pattern Recogn Lett 2005;23:459-70.

[13] ETDRSReportNumber10,Gradingdiabeticretinopathyfro mstereoscopic color fundus photographs - an extension of the modified Airlie house classification. Ophthalmology 1991;98:783-803.

[14] Davis MD, Bressler SB, Aiello LP, Bressler NM, Browning DJ, Flaxel CJ, et al. Comparison of timedomain oct and fundus photographic assessments of retinal thickening in eyes with diabetic macular edema. Invest Ophthalmol Vis Sci 2008;49:1745-52.
[15] Gonzales RC, Woods RE. Digital image processing. New York: AddisonWesley; 1992. p. 75-140.

[16] Sinthanayothin C, Boyce JF, Cook HL, Williamson TH. Automated localization of the optic disc, fovea, and retinal blood vessels from digital colour fundus images. Br J Ophthalmol 1999;82:902-10.

[17] Walter T, Klein JC, Massin P, Erginay A. A Contribution of image processing to the diagnosis of diabetic retinopathy-detection of exudates in colour fundus images of the human retina. IEEE Trans Med Imag 2002;21:1223-42.

[18] Sanchez CI, Hornero R, Lopez MI. Retinal image analysis to detect and quantify lesionsassociated withdiabeticretinopathy .In: Proceedingsofthe International Conference on Engineering in Medicine and Biology Society IEEE. 2004. p. 1324-7.

[19] Zhang X, Opas C. Detection and classification of bright lesions in colour fundus images. In: Proceedings of the International Conference on Image Processing IEEE. 2004. p. $129-42$.

[20] JavittJC,AielloLP,BassiLJ,ChiangEP,CannerJK,KleinR . Detectingand treatingretinopathyinpatientswithtypeIdiabetesmellitus.S avingsassociated with improved implementation of current guidelines. Ophthalmology 1991;98:1535-72.

[21] Acharya, U. R., Lim, C. M., Ng, E. Y. K., Chee, C., and Tamura, T., Computer based detection of diabetes retinopathy stages using digital fundus images. J. Eng. Med. 223(H5):545-553, 2009.

[22] [Raju Maher, Dr.Mukta Dhopeshwarkar "Automated Detection of Non-proliferative Diabetes Retinopathy Using Fundus Images" International Journal of Advanced Research in Computer Science and Software Engineering Volume 5, Issue 3, March 2015.

[23] Raju Sahebrao Maher, Sangramsing N. Kayte, Sandip T. Meldhe, Mukta Dhopeshwarkar, "Automated Diagnosis Non-proliferative Diabetic Retinopathy in Fundus Images using Support Vector Machine" International Journal of Computer Applications (0975 - 8887)Volume 125 - No.15, September 2015.

[24] Raju Sahebrao Maher, Dnyaneshwar S. Panchal, Sangramsing Kayte, Dr. Mukta Dhopeshwarkar" Automatic Identification of Varies Stages of Diabetic Retinopathy Using Retinal Fundus Images" International Journal of Advanced Research in Computer Science and Software Engineering, Volume 5, Issue 9, September 2015 .

[25] Raju Maher, Sangramsing Kayte, Dnyaneshwar Panchal, Pankaj Sathe, Sandip Meldhe, "A Decision Support System for Automatic Screening of Non-proliferative Diabetic Retinopathy" International Journal of Emerging Research in Management and Technology, Volume4,Issue-10, October-2015. 